\title{
Information Retrieval from Emotions and Eye Blinks with help of Sensor Nodes
}

\author{
Puneet Singh Lamba ${ }^{1}$, Deepali Virmani ${ }^{2}$ \\ ${ }^{1}$ University School of Information, Communication and Technology, GGSIPU, India \\ ${ }^{2}$ Department of Computer Science, Bhagwan Parshuram Institute of Technology, GGSIPU, India
}

\begin{tabular}{l} 
Article Info \\
\hline Article history: \\
Received Feb 2, 2018 \\
Revised Apr 30, 2018 \\
Accepted May 17, 2018 \\
\hline
\end{tabular}

Keyword:

Emoticons

Emotions

Eye blink

Information Retrieval

Sensor networks

\begin{abstract}
In everyday life, there are situations where the only way to communicate are emotions. EMOTICONS are the epitome of the same. This aspect of communication can also be used in emergency situations (terrorist attacks, hijacks) where the only way to communicate is by performing some extraordinary actions or through some emotions. Incorporating technology to the above mentioned circumstances the paper proposes a novel framework of detecting an emergency situation by retrieving information from emotions and eye blinks using sensor nodes. The proposed framework can be deployed in places (hotels, banks, airports etc.) which are more suspected to attacks. The framework takes input from real time parameters: eye blinks, emotions, heart rate. Based on behavioral changes, biological changes and physical changes the proposed framework extracts meticulous information. The proposed framework is further validated through implementation of a facial emotion recognition system that successfully recognizes various human emotions. The facial emotion recognition system of the proposed framework is compared with existing SVM technique in terms of accuracy, training and testing error. Accuracy with the proposed system is increased to $78.40 \%$ in comparison with existing SVM that is $75.37 \%$ and the training error is decreased to 0.004103 whereas with the existing SVM method training error is 0.008935 .
\end{abstract}

Copyright $\odot 2018$ Institute of Advanced Engineering and Science. All rights reserved.

\section{Corresponding Author:}

Puneet Singh Lamba,

University School of Information, Communication and Technology,

Guru Gobind Singh Indraprastha University,

Sector 16 C, Kakrola, New Delhi, Delhi 110078, India.

Mobile: 09810907138

Email: singhs.puneet@gmail.com.

\section{INTRODUCTION}

In recent times many events of hostages and hijacks have happened and there is an urgent need of an automated system which can make the concerned agencies aware of the situation in time and thus help them to take necessary action. Wireless Sensor Networks can be used in attack prone areas to examine the situation and hence will help the concerned agencies to take appropriate action in time.

The 26/11 Mumbai attacks were a planned series of attacks, when a group of 10-12 terrorists of Lashkar-e-Taiba, carried out a sequence of coordinated bombing and shooting that continued for four days [1], [2]. Densely populated areas like railway station and 5 star hotels (Hotel Taj) were their main targets. The attacks, which were heavily censured globally, began on $26^{\text {th }}$ November and lasted until $29^{\text {th }}$ November 2008, killing 164 people and wounding at least 300. A lot of damage had already been done even before the security agencies came to know what actually had happened. So if there would have been such automated systems, a lot would have been saved in terms of lives and public property. 
Another such event of hostage includes hijacking of Indian Airline Flight 814 en route from Tribhuvan International Airport, Nepal to Indira Gandhi Airport, India on Friday, 24 December 1999. The most recent Uri attack was an inhuman attack by heavily armed terrorists in Uri area of Baramulla district, Jammu and Kashmir. It is considered as "the deadliest attack on security forces in last two decades". No terrorist group has claimed responsibility for the same.

In this paper we propose a novel framework for detecting an emergency situation which uses the intelligent information retrieved from pulse rate and eye blinks [3] using wireless sensor nodes as well as behavioral patterns [4], [5] exhibited by humans in response to any changes in the former parameters. To the best of our knowledge, such security framework that confirms emergency or intrusion detection on all given parameters does not exist.

The parameters in response abnormal situation discussed here are:

a. Eye blink pattern,

b. Human Emotions,

c. Heart rate

For each parameter we intent to analyze the behavioral (emotion), biological (pulse rate) and physical (eye blink) pattern changes as measured by the systems deployed which can in future be used to install an emergency or intrusion warning system.

Author in [6] have presented a novel approach to detect whether the eyes in an image (still) are closed or not and this approach has wide ranging applications in facial expression recognition, fatigue detection, and so on. A similar analysis in [7] has been discussed to detect eye blinks in a video sequence from a standard camera. Emotion is also one of the parameters which play an important part in our proposed framework. In [8] a regional hidden Markov model (RHMM) is proposed for recognizing facial expressions in video sequences. Facial action units are described for eyebrows, eyes and mouth regions by RHMMs. Proposed technique has outperformed other existing methods when tested with extended Cohn-Kanade database. Author in [9] presents an Automatic Face Analysis (AFA) system to inspect facial expressions taking into consideration both permanent facial features (brows, eyes, mouth) and momentary facial features (deepening of facial furrows).

\section{RESEARCH METHOD}

Sensor Nodes as known for the monitoring abilities such as accuracy and sensitivity are used as the baseline for the proposed framework. Figure 1 describes the proposed Framework.

The intelligent information collected from standalone parameters, will act as input to the system which after performing calculations will decide whether it has crossed the threshold or not. Upon crossing the threshold an alarm or buzzer or some message will be transmitted to the concerned authorities. The sensors nodes will be deployed to extract the profound information from the given parameters, thus can be used as forerunner for provoking the alarm messages.

a. Eye blink

This attribute is of utmost importance as far as our proposed framework is concerned. Average eye blinking rate is around 12-19 per minute. (Intentional Eye blinks $>25 \rightarrow$ Abnormal).

Proposed framework uses the sensor placed in the line of sight of the eye which continuously studies the blink pattern. Sensors used will draw a curve to indicate a change in eye blink [10], [11] rate.

b. Human emotions

Emotion is a cognitive state that is generated impulsively rather than through responsive effort and is accompanied by physiological changes which are familiar to the outer world. Some emotions which humans face in everyday life are worry, surprise, confuse, happy etc. Human vision can practice emotion as coupled with personality, temperament, and mood. Computer Vision tries to follow the human vision by analyzing digital image as input. Sensors deployed will continuously study the human emotions. A database of emotions with an instance of time will be maintained with sensor nodes.

c. Heart rate

Heart rate is the number of times a heart beats in a minute. Heart rate [12] varies from person to person depending on one's age, body size, fitness and heart condition. The same also depends on whether the person is running, standing, sitting, moving or is under influence of some medication. Emotions can also increase the heart rate. A healthy heart beats 60 to 100 times in a minute and can spike up due to stress, overexertion or nervousness. Our proposed framework will continuously monitor the heart rate.

The proposed framework unfolds the entire structure of the process combined together. Smart motes (nodes) will be installed as per the requirements. Three types of sensors (eye blink, emotion detection and heart rate sensors) will be deployed. The sensors will work independently and will transfer the sensed attributes to the smart server via smart gate where the actual feature selection will take place. Then after 
some observation, calculation and comparisons, the final verdict (whether the threshold is reached or not) will be taken. If threshold is crossed, alarm will be raised. For example, if the eye blink sensor senses an unusual blink pattern or number, in addition to a Fear or Surprise emotion detected by the emotion detection sensor or an abnormal heart rate, the situation is an alarming one. Blink pattern and emotions can be calculated by still or moving images via specialized cctv's. For heart rate concerned persons (bar tenders in hotels, bank managers, flight attendants) will be equipped with wireless heart rate sensors or straps.

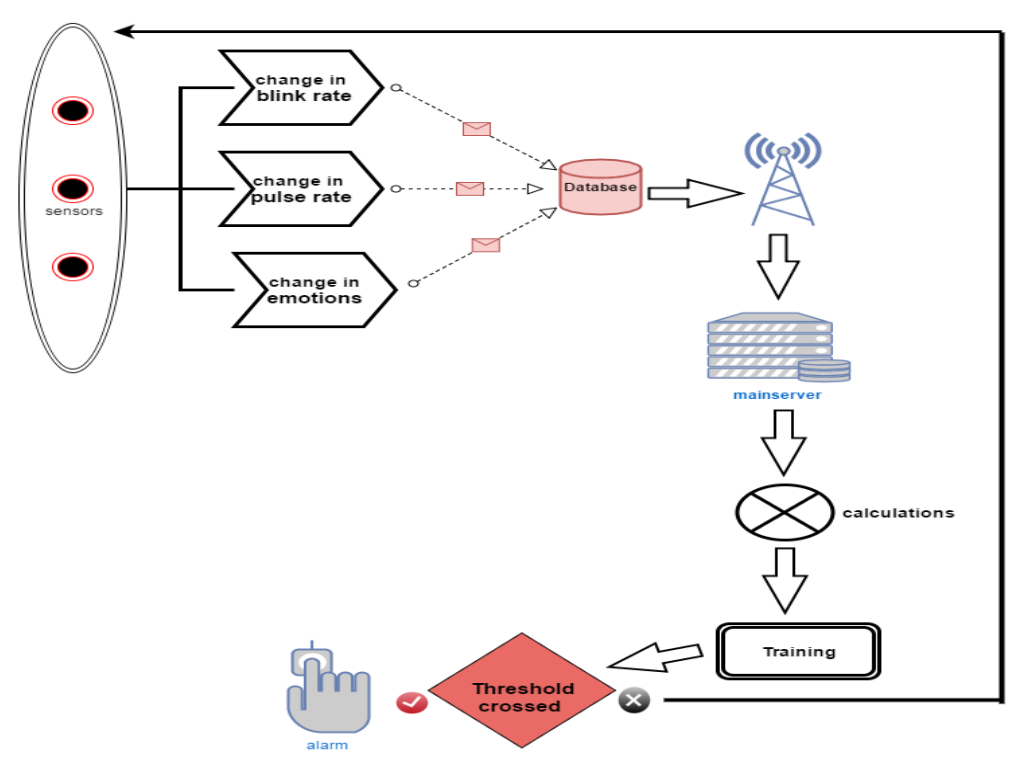

Figure 1. Proposed Framework for Exigency detection

The proposed framework works in two phases. The first phase (analysis phase) takes input from eye blinks, emotions and heart rate. The second phase constitutes the classification phase. The reactions could be change in behavior or emotions or physiological over given time. In this paper a Perspicacious Information Retrieval technique which senses serious diversion of parameters from baseline values via sensors spanned across target zones is presented. The data is transmitted to a base station which is further processed at main server. The moment major deflection from normal is observed using the information gathered, sensors are trained to issue alarm signals as in when such abnormal activity is sensed.

\subsection{Implementing information retrieval technique}

Figure 2 shows how methodically Perspicacious Information Retrieval Technique (PIRT) is implemented at servers where messages stating unusual human emotion, pulse rate and blink pattern are received at base station. It is important to know that input values would be gathered from some trained staff (air hostess in airplanes, bartenders in hotels, some officials in banks etc) having the knowledge of how to react in an emergency situation.

The proposed framework should be deployed in areas which are in direct line of sight of the concerned persons and will help generate alarm signals as soon as change in behavioral or blink patterns is sensed. Messages acquired are further refined in order to excerpt intelligent information from it. Figure 2 presents a novel prognostic algorithm which takes as input sensed data from the preceding stages and figure out certain conditions on the data received.

Various stages of PIRT are:

a. Z1: Eye blink

This standalone condition evaluates the Blink pattern (BP) and hence the numbers of Blinks in say 1 minute. Average eye blinking rate is around 12-19 per minute. So an unusual number/pattern will be the indication that something abnormal is there. For a precise decision we are taking it 25 Blinks per minute. The value stated which approximately accounts to 25 is the lowest default value. As no signs of change in blink pattern are observed below value - No alarm will be raised. For getting the BRM (blink rate per minute), frames from the video sequence can be extracted and will be further processed for detecting face and hence the eye portion of the image. From the eye landmarks [7] detected from the image, the eye aspect ratio (EAR) that is used as an approximate of the eye opening/closing state can be calculated. The eye aspect ratio (EAR) 
is computed between height and width of the eye. Characteristic ratio of the open eye has a small discrepancy among humans and it is fully uniform to a constant scaling of the image. As blinking is practiced by both eyes simultaneously, the EAR of the pair is averaged. The EAR is primarily constant for an open eye and is approximately zero for a closed eye.

Pattern for condition Z1:

\section{\{ Z1: calculate BRM}

if $(\mathrm{BRM}<25)$

no alert ;

Else

proceed to $\mathrm{Z} 2$ ( $2^{\text {nd }}$ condition) $\}$
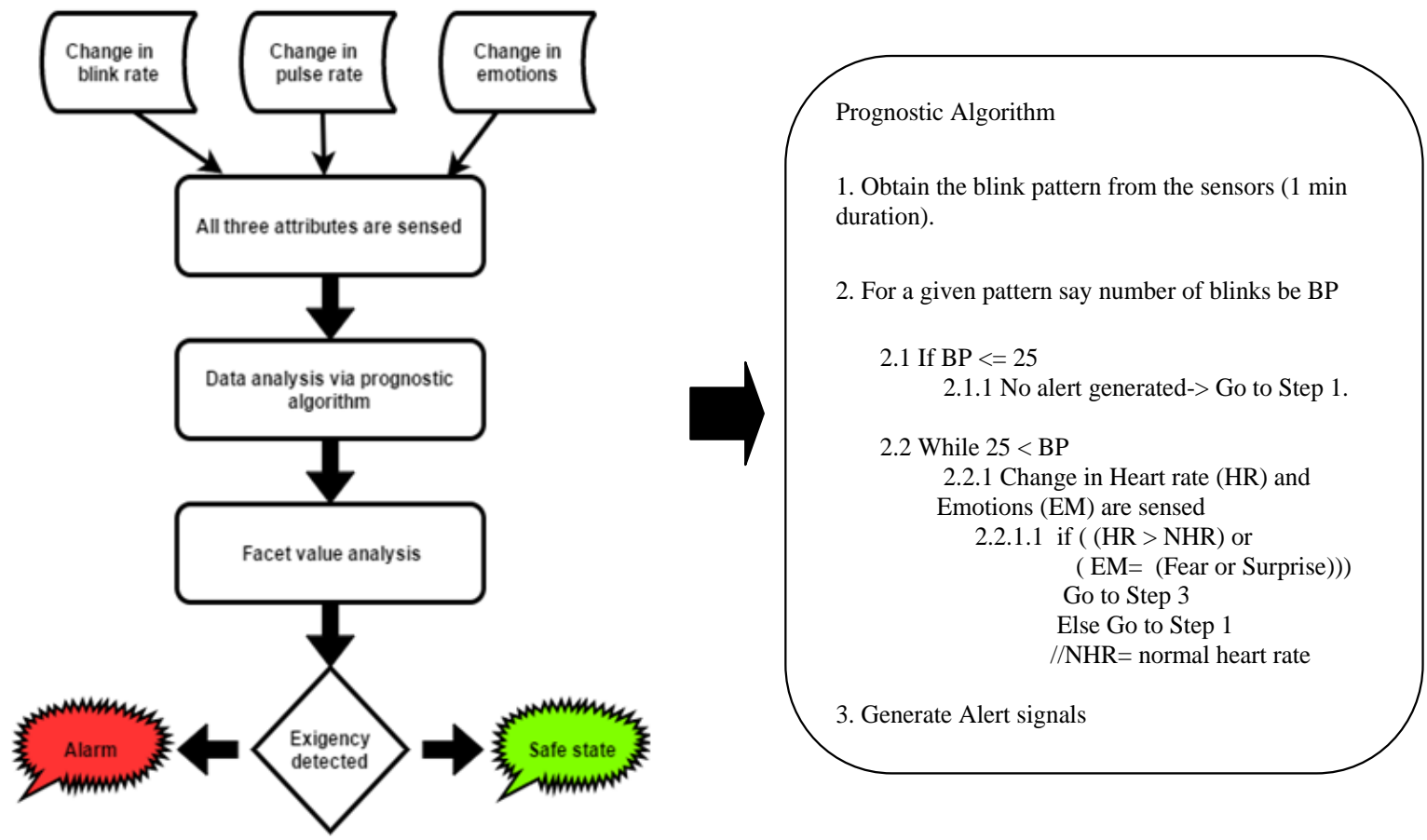

Figure 2. Perspicacious Information Retrieval Technique for exigency detection

b. Z2: Heart rate

This condition will be evaluated only if condition $\mathrm{Z} 1$ is true and will evaluate whether the Heart rate (HR) or pulse has crossed the normal defined rate. If it has, alarm will be generated; otherwise we proceed to check the next condition which is getting ORed with the current condition.

Pattern for condition Z2:

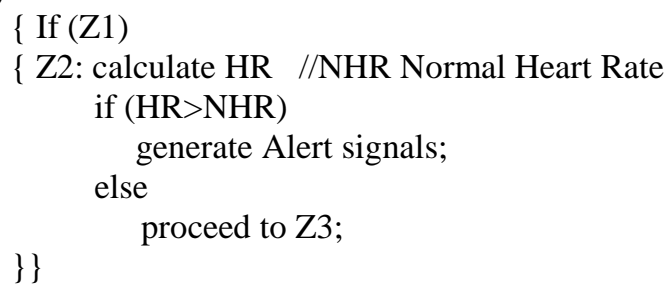

c. Z3: Facial emotion

This condition evaluates the emotion detected. If the resultant emotion detected is FEAR or SURPRISE, alarm signal will be generated (only if condition Z1 stands true) else we will proceed to first condition again. It is important to note that either Condition 2 (Z2), 3 (Z3) or both should be tested to generate warning messages (given $\mathrm{Z} 1$ is true).

Pattern for condition Z3:

If $(\mathrm{Z} 1)$

\{Z3: Detect Emotion 


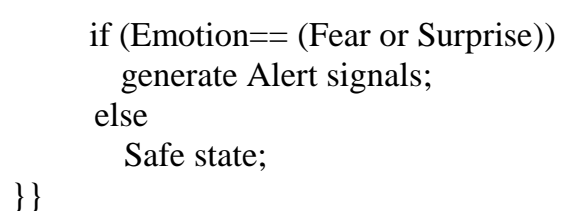

\subsection{Facet value analysis}

The facet evaluation is done on the sensed data to further figure out conditions and generate alert signals whenever condition $\mathrm{Z} 1, \mathrm{Z} 2$ or $\mathrm{Z} 3$ evaluates to true/false in certain permutations or combination. Based on above analysis we validate a framework that can hatch alert signals based on messages received by the sensors deployed. Figure 3 shows some cause effect graphs which state that condition Z1 in conjunction with $\mathrm{Z} 2$ or $\mathrm{Z} 3$ or both is a necessary prerequisite for the framework to issue warning signal.

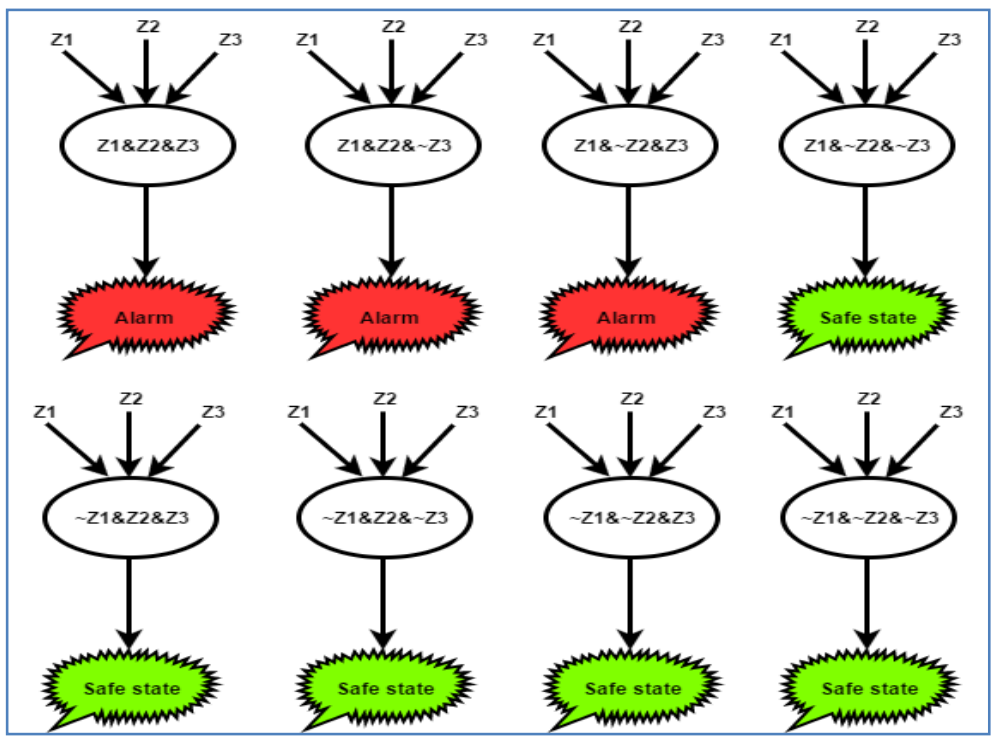

Figure 3. Cause graph based on condition Evaluated

\subsection{Emotion detection}

The paper presents a detailed analysis of one of the parameters of the proposed framework: Human Emotions [13], [14]. A Facial Emotion Recognition System is proposed that would recognize 7 emotions labeled Neutral, Angry, Disgust, Fear, Happy, Sad and Surprise using Matlab. The proposed system will extract emotions on the basis of some unique features. Figure 4 presents a novel algorithm for detecting emotions from an image. The image to be tested can be taken from a smart phone using an app IP Webcam which turns the android phone into wireless camera.

\section{RESULTS AND DISCUSSION}

The performance of proposed Facial emotion recognition system is evaluated and Table 1 showcases the comparison between the proposed method and existing SVM during the training and testing phase. The proposed method is compared with existing SVM (support vector machine) technique in terms of accuracy, training and testing error. Accuracy and training error comparison between SVM and the proposed method is shown in Figure 5. Accuracy with the proposed method is increased to $78.40 \%$ in comparison with existing SVM that is $75.37 \%$. Training error for the proposed method is decreased to 0.004103 whearas with the existing SVM method training error is 0.008935 . 


\section{Algorithm for Emotion Detection}

- $\quad$ Preprocess the database (cohn-kanade)

Create train directory

mkdir('Training_datal0');

mkdir('Training_datal5');

mkdir('Training_datal6');

folder $0:=$ all neutral images

folder $1:=$ all angry images

folder $6:=$ all surprise images

- Test directory $<=(25-30 \%$ images of train directory)

- $\quad$ Training and testing data matrix for concerned database is prepared using local binary pattern.

for $\mathrm{i}=1$ :no_files

filename $=$ files $\{i\}$;

$\mathrm{Im}=$ imread(filename);

Call next two steps for face detection, cropping (eye, nose and mouth) and local binary histogram [15].

trainX(i,:) = lbpHist;

trainY(i,str2double(filename(19))+1) $=1$

Proceed to Training step.

- Detect faces from the images using voila jones algorithm [16] [17].

$[\mathrm{X}, \mathrm{Y}$, height, width $]=$ violaJones $(\operatorname{Image}) ;$

- $\quad$ The target face will be divided into three regions (eye, nose and mouth)

cropL = imcrop(Image, $[X, Y$, height, width $]$ );

cropL = imresize (cropL, $[40,40])$;
$\mathrm{L} 1=\operatorname{cropL}(10: 20,10: 30) ; / /$ eye

$\mathrm{L} 2=\operatorname{cropL}(20: 30,10: 30) ; / /$ nose

$\mathrm{L} 3=\operatorname{cropL}(30: 40,10: 30) ; / /$ mouth

$\mathrm{lbpH} 1=\mathrm{lbp}(\mathrm{L} 1) ; / /$ local binary pattern (eye)

$\mathrm{lbpH} 2=\mathrm{lbp}(\mathrm{L} 2) ; / /$ local binary pattern (nose)

lbpH3 = lbp(L3); //local binary pattern (mouth)

lbpHist=[lbpH1,lbpH2,lbpH3];//LBP

//A histogram of the labeled image $\mathrm{f}_{1}(\mathrm{x}, \mathrm{y})$ can be defined as

$\mathrm{H}_{\mathrm{i}}=\sum_{\mathrm{x}, \mathrm{y}} \mathrm{I}\left\{\mathrm{f}_{\mathrm{l}}(\mathrm{x}, \mathrm{y})=\mathrm{i}\right\}, \mathrm{i}=0, \ldots, \mathrm{n}-1$

in which $\mathrm{n}$ is the number of different labels produced by the LBP operator and

$\mathrm{I}\{\mathrm{A}\}=1, \mathrm{~A}$ is true

$$
=0, \mathrm{~A} \text { is false. }
$$

This histogram contains information about the distribution of the local micro patterns, such as edges, spots and flat areas, over the whole image.

- $\quad$ (Training step) Train matrices are then used to train the classifier (neural network). Network is trained by applying training data and training parameters (learning rate parameter (eta $=0.01$ ) and number of epochs (nEpochs $=500$ ), hidden layers $(H=16)$ ) as input which results in updated weights of the input and hidden layer with minimum error

- To complete the emotion detection, practice the proposed system takes as input updated weights $\mathrm{w}$ and $\mathrm{v}$ (backward pass) from previous step and testing points X (LBP features of image to be tested) and concludes with the family of the data as anticipated by the network.

Figure 4. Algorithm for detecting emotions

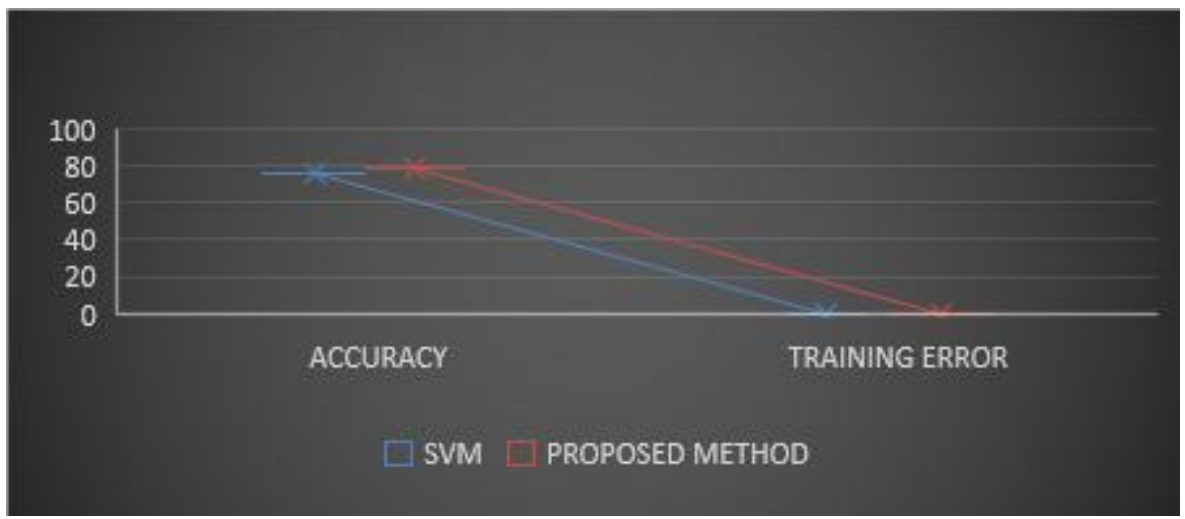

Figure 5. Accuracy and training error comparison between SVM and the Proposed Method

Figure 6 shows the image which system accepts as input and Figure 7 depicts the resultant emotion (neutral) being detected. Two different resultant emotions (surprise, happy) are also shown in Figure 8 and Figure 9. Training error is shown in Figure 10 which reduces with each iteration. 
Table 1. Comparison between Proposed Method and SVM

\begin{tabular}{lllll}
\hline & SVM (Training) & $\begin{array}{l}\text { Proposed Method } \\
\text { (Training) }\end{array}$ & $\begin{array}{l}\text { SVM } \\
\text { (Testing) }\end{array}$ & $\begin{array}{l}\text { Proposed Method } \\
\text { (Testing) }\end{array}$ \\
\hline Total Number of Instances & 370 & 370 & 264 & 264 \\
Correctly Classified Instances & 370 & 369 & 199 & 207 \\
Incorrectly Classified Instances & 0 & 1 & 65 & 57 \\
Mean absolute error & 0.2041 & 0.0025 & 0.2098 & 0.0722 \\
Kappa statistic & 1 & 0.9962 & 0.6321 & 0.6791 \\
Root mean squared error & 0.3006 & 0.0229 & 0.3099 & 0.2281 \\
Relative absolute error & $100.4048 \%$ & $1.2529 \%$ & $106.4637 \%$ & $36.6319 \%$ \\
Root relative squared error & $94.4743 \%$ & $7.1946 \%$ & $100.5206 \%$ & $73.994 \%$ \\
\hline
\end{tabular}

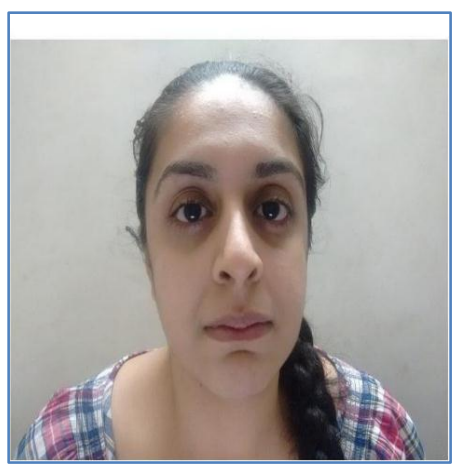

Figure 6. Input image

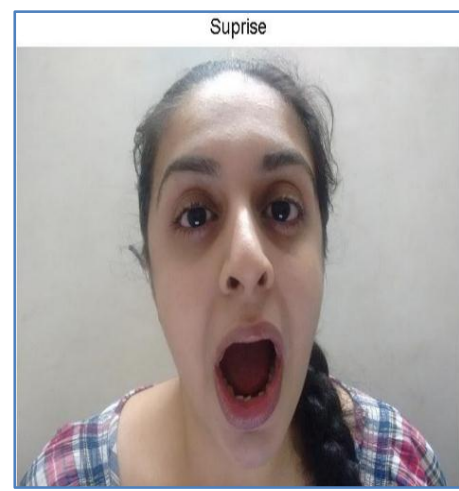

Figure 8. Emotion 2

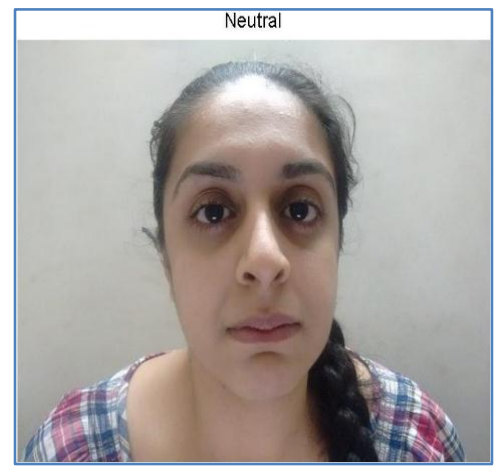

Figure 7. Emotion 1

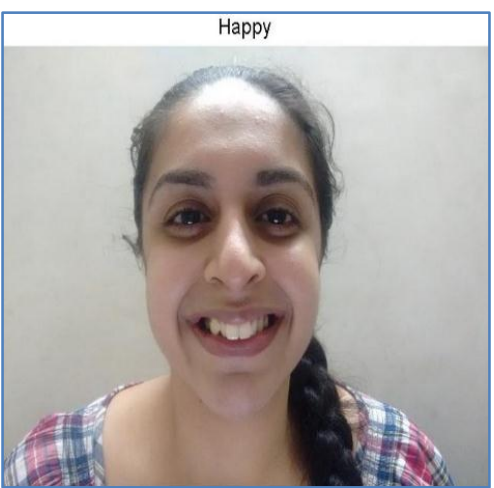

Figure 9. Emotion 3

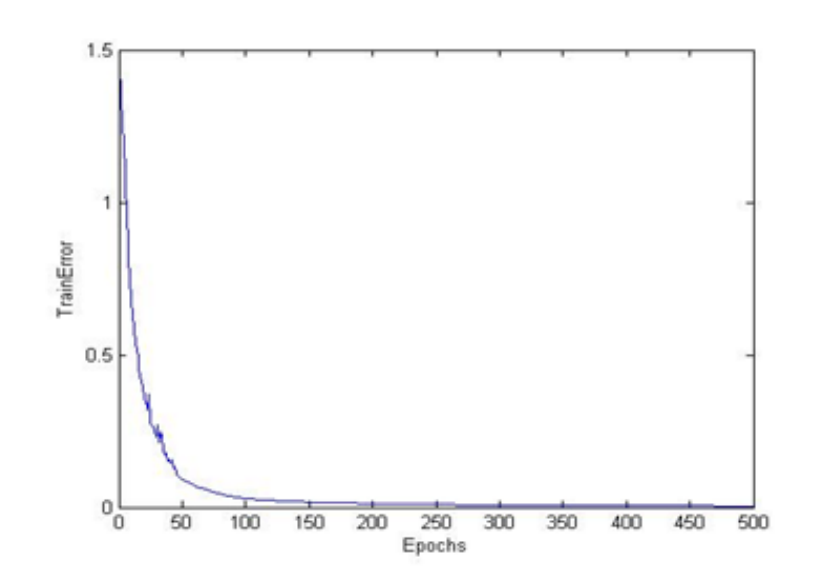

Figure 10. Training error 


\section{CONCLUSION}

This paper underlines measurable parameters that can be perceived using sensor networks in terms of intelligent information messages. Such parameters include eye blink pattern, emotions and heart rate, of which blink pattern is given the utmost importance. Resultant messages are then percolated and conditionally tagged to excerpt intelligent information, thus generating exigency alert. Also it highlights both the sufficient and necessary conditions required for alarm to be raised. Paper proposes an intelligent framework along with Perspicacious Information Retrieval Technique and the prognostic algorithm for detecting the exigency. The proposed Facial Emotion Recognition System recognizes 7 emotions labeled Neutral, Angry, Disgust, Fear, Happy, Sad and Surprise. The algorithm is validated through MATLAB and results depict the categorization of faces as neutral, surprise and happy depending on the expression.

\section{REFERENCES}

[1] Magnier, Mark; Sharma, Subhash (27 November 2008), "India terrorist attacks leave at least 101 dead in Mumbai," Los Angeles Times. p. A1. Retrieved 28 November 2008.

[2] Friedman, Thomas (17 February 2009), "No Way, No How, Not Here," The New York Times. Archived from the original on 29 August 2012. Retrieved 17 May 2010.

[3] Mr. Raees Ahmad, Prof. J.N. Borole, "Drowsy Driver Identification Using Eye Blink detection", International Journal of Computer Science and Information Technologies, 2015, vol. 6, no. 1, pp. 270-274.

[4] Kusrini Kusrini, M. Dedi Iskandar, Ferry Wahyu Wibowo, "Multi Features Content-Based Image Retrieval Using Clustering And Decision Tree Algorithm", TELKOMNIKA (Telecommunication, Computing, Electronics and Control), 2016, vol. 14, no. 2, pp. 1480-1492.

[5] Jinhua Zhang, Daniel Scholten, "A Face Recognition Algorithm Based on Improved Contourlet Transform and Principle Component Analysis”, TELKOMNIKA (Telecommunication, Computing, Electronics and Control), 2016, vol. 14, no. 2A, pp. 114-119.

[6] Fengyi Song, Xiaoyang Tan, Xue Liu, Songcan Chen, "Eyes closeness detection from still images with multi-scale histograms of principal oriented gradients", Pattern Recognition, Elsevier, 2014, vol. 47, no. 9, pp. 2825-2838.

[7] TerezaSoukupova and Jan Cech, "Real-Time Eye Blink Detection using Facial Landmarks", Proceedings of the 21 st Computer Vision Winter Workshop Rimske Toplice, Slovenia, 2016.

[8] Y. Sun and A.N. Akansu, "Facial expression recognition with regional hidden Markov models", ELECTRONICS LETTERS, 2014, vol. 50, no. 9, pp. 671-673.

[9] Ying-li Tian, Takeo Kanade, and Jeffrey F. Cohn, "Recognizing Action Units for Facial Expression Analysis", IEEE Transactions on Pattern Analysis and Machine Intelligence, 2001, vol. 23, no. 2, pp. 97-115.

[10] J. Cech, V. Franc, J. Matas, "A 3D approach to facial landmarks: Detection, refinement, and tracking”, In Proc. International Conference on Pattern Recognition, 2014, pp. 2173-2178.

[11] Rehab F. Abdel-Kadern, Randa Atta, Sheren El-Shakhabe, "An efficient eye detection and tracking system based on particle swarm optimization and adaptive block-matching search algorithm", Engineering Applications of Artificial Intelligence, 2014, vol. 31, pp. 90-100.

[12] H. Chuduc, K. Nguyenphan, and D. Nguyen, "A Review of Heart Rate Variability and its Applications", APCBEE Procedia ELSEVIER, 2013, vol. 7, pp. 80-85.

[13] Ching Yee Yong, Rubita Sudirman, Kim Mey Chew, "Colour Perception on Facial Expression towards Emotion", TELKOMNIKA (Telecommunication, Computing, Electronics and Control), 2012, vol. 10, no. 4, pp. 723-733.

[14] Yutai Wang, Xinghai Yang, Jing Zou, "Research of Emotion Recognition Based on Speech and Facial Expression. TELKOMNIKA (Telecommunication, Computing, Electronics and Control), 2013, vol. 11, no. 1, pp. 83-90.

[15] Timo Ahonen, Abdenour Hadid, and Matti Pietikainen, "Face Recognition with Local Binary Patterns", ECCV 2004, LNCS 3021, 2004, pp. 469-481.

[16] Paul Viola and Michael J. Jones, "Robust Real-Time Face Detection", International Journal of Computer Vision, 2004, vol. 57, no. 2, pp. 137-154.

[17] Yi-Qing Wang, "An Analysis of the Viola-Jones Face Detection Algorithm”, Image Processing On Line, 2014, vol. 4, pp. 128-148.

\section{BIOGRAPHIES OF AUTHORS}

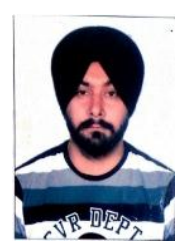

Puneet Singh Lamba received the Master degree (M.tech) in Information Technology from University School of Information And Communication Technology, Guru Gobind Singh Indraprastha University in 2013. He is a PhD candidate in Information Technology at USICT, GGSIPU. His research interests are in the areas of Sensor Networks and Adhoc Networks. He has more than 8 years of teaching experience and is currently working as Assistant Professor in Bharati Vidyapeeth's College of Engineering New Delhi, India. 
Dr. Deepali Virmani received the doctoral degree in Computer Science from Netaji Subhas Institute of Technology, New Delhi in 2012. Her research interests are in the areas of Sensor Networks, Data Mining and Security. She has published more than 60 papers in referred International journals and conferences. She is branch counselor IEEE student chapter and CSI student branch and has more than 15 years of teaching experience. She is currently working as Associate Professor (Head of Computer Science Department) in Bhagwan Parshuram Institute of Technology approved by Guru Gobind Singh Indraprastha University, New Delhi, India. 